\title{
Perturbations Around Black Holes
}

\author{
Bin Wang \\ Department of Physics, Fudan University, Shanghai 200433, China
}

(Received on 24 November, 2005)

\begin{abstract}
Perturbations around black holes have been an intriguing topic in the last few decades. They are particularly important today, since they relate to the gravitational wave observations which may provide the unique fingerprint of black holes' existence. Besides the astrophysical interest, theoretically perturbations around black holes can be used as testing grounds to examine the proposed AdS/CFT and dS/CFT correspondence.
\end{abstract}

\section{INTRODUCTION}

Present astronomical searching for black holes are based on the study of images of strong X-rays taken of matter around the probable black hole candidate. Usually the astronomers study the X-ray binary systems, which consist of a visible star in close orbit around an invisible companion star which may be a black hole. The companion star pulls gas away from the visible star. As this gas forms a flattened disk, it swirls toward the companion. Friction caused by collisions between the particles in the gas heats them to extreme temperatures and they produce X-rays that flicker or vary in intensity within a second. Many bright X-ray binary sources have been discovered in our galaxy and nearby galaxies. In about ten of these systems, the rapid orbital velocity of the visible star indicates that the unseen companion is a black hole. The X-rays in these objects are produced by particles very close to the event horizon. In less than a second after they give off their X-rays, they disappear beyond the event horizon. In a very recent study, the sharpest images ever taken of matter around the probable black hole at the centre of our Galaxy have brought us within grasp of a crucial test of general relativity $\nmid$ a picture of the black hole's 'point of no return' [1]. A supermassive black hole has been proved existing in the centre of our own Galaxy.

However this way of searching for black hole is not direct, to some sense it relies on the evolution behavior of the visible companion. Whether a black hole itself has a characteristic 'sound', which can tell us its existence, is a question we want to ask. Performing numerical studies of perturbations around black holes, it was found that during a certain time interval the evolution of the initial perturbation is dominated by damped single-frequency oscillation. The frequencies and damping of these oscillations relate only to the black hole parameters, not to initial perturbations. This kind of perturbation which is damped quite rapidly and exists only in a limited time interval is referred to as the quasinormal modes. They will dominate most processes involving perturbed black holes and carry a unique fingerprint which would lead to the direct identification of the black hole existence. Detection of these quasinormal modes is expected to be realized through gravitational wave observations in the near future. In order to extract as much information as possible from gravitational wave signal, it is important that we understand exactly how the quasinormal modes behave for the parameters of black holes in different models. (see [2][3] for a review and references therein)

Besides the astronomical interest, the perturbations around black holes have profound theoretical implications. Motivated by the discovery of the correspondence between physics in the Anti-de Sitter (AdS) spacetime and conformal field theory (CFT) on its boundary (AdS/CFT), the investigation of QNM in AdS spacetimes became appealing in the past several years. It was argued that the QNMs of AdS black holes have direct interpretation in term of the dual CFT (for an extensive but not exhaustive list see [4][5][6][7][8, 9][10-15]) . In de Sitter (dS) space the relation between bulk dS spacetime and the corresponding CFT at the past boundary and future boundary in the framework of scalar perturbation spectrums has also been discussed [16, 17][18][19]. A quantitative support of the $\mathrm{dS} / \mathrm{CFT}$ correspondence has been provided. More recently the quasinormal modes have also been argued as a possible way to detect extra dimensions [20].

The study of quasinormal modes has been an intriguing subject. In this review we will restrict ourselves to the discussion of non-rotating black holes. We will first go over perturbations in asymptotically flat spacetimes. In the following section, we will focus on the perturbations in AdS spacetimes and $\mathrm{dS}$ spacetimes and show that quasinormal modes around black holes are testing grounds of AdS/CFT and dS/CFT correspondence. We will present our conclusions and outlook in the last part.

\section{PERTURBATIONS IN ASYMPTOTICALLY FLAT SPACETIMES}

A great deal of effort has been devoted to the study of the quasinormal modes concerned with black holes immersed in an asymptotically flat spacetime. The perturbations of Schwarzschild and Reissner-Nordstrom (RN) black holes can be reduced to simple wave equations which have been examined extensively [2][3]. However, for nonspherical black holes one has to solve coupled wave equations for the radial part and angular part, respectively. For this reason the nonspherical case has been studied less thoroughly, although there has recently been progress along these lines [21]. In asymptotically flat black hole backgrounds radiative dynamics always proceeds in the same three stages: initial impulse, quasinormal ringing and inverse power-law relaxation.

Introducing small perturbation $h_{\mu v}$ on a static spherically symmetric background metric, we have the perturbed metric with the form

$$
g_{\mu v}=g_{\mu v}^{0}+h_{\mu v}
$$


In vacuum, the perturbed field equations simply reduce to

$$
\delta R_{\mu \nu}=0
$$

These equations are in linear in $h$.

For the spherically symmetric background, the perturbation is forced to be considered with complete angular dependence. From the 10 independent components of the $h_{\mu v}$ only $h_{t t}, h_{t r}$, and $h_{r r}$ transform as scalars under rotations. The $h_{t \theta}, h_{t \phi}, h_{r \theta}$, and $h_{r \phi}$ transform as components of two-vectors under rotations and can be expanded in a series of vector spherical harmonics while the components $h_{\theta \theta}, h_{\theta \phi}$, and $h_{\phi \phi}$ transform as components of a $2 \times 2$ tensor and can be expanded in a series of tensor spherical harmonics [2][3]. There are two classes of tensor spherical harmonics (polar and axial). The differences are their parity under space inversion $(\theta, \phi) \rightarrow(\pi-\theta, \pi+\phi)$. After the inversion, for the function acquiring a factor $(-1)^{l}$ refers to polar perturbation, and the function acquiring a factor $(-1)^{l+1}$ is called the axial perturbation.
The radial component of a perturbation outside the event horizon satisfies the following wave equation,

$$
\frac{\partial^{2}}{\partial t^{2}} \chi_{\ell}+\left(-\frac{\partial^{2}}{\partial r_{*}^{2}}+V_{\ell}(r)\right) \chi_{\ell}=0
$$

where $r_{*}$ is the "tortoise" radial coordinate. This equation keeps the same form for both the axial and polar perturbations. The difference between the axial and polar perturbations exists in their effective potentials. For the axial perturbation around a Schwarzschild black hole, the effective potential reads

$$
V_{\ell}(r)=\left(1-\frac{2 M}{r}\right)\left[\frac{\ell(\ell+1)}{r^{2}}+\frac{2 \sigma M}{r^{3}}\right] .
$$

However for the polar perturbation, the effective potential has the form

$$
V_{\ell}(r)=\left(1-\frac{2 M}{r}\right) \frac{2 n^{2}(n+1) r^{3}+6 n^{2} M r^{2}+18 n M^{2} r+18 M^{3}}{r^{3}(n r+3 M)^{2}} .
$$

Apparently these two effective potentials look quite different, however if we compare them numerically, we will find that they exhibit nearly the same potential barrier outside the black hole horizon, especially with the increase of $l$. Thus polar and axial perturbations will give us the same quasinormal modes around the black hole.

Solving the radial perturbation equation here is very similar to solving the Schrodinger equation in quantum mechanics. We have a potential barrier outside the black hole horizon, and the incoming wave will be transmitted and reflected by this barrier. Thus many methods developed in quantum mechanics can be employed here. In the following we list some main results of quasinormal modes in asymptotically flat spacetimes obtained before.

(a) It was found that all perturbations around black holes experience damping behavior. This is interesting, since it tells us that black hole solutions are stable.

(b) The quasinormal modes in black holes are isospectral. The same quasinormal frequencies are found for different perturbations for example axial and polar perturbations. This is due to the uniqueness in which black holes react to perturbations.

(c) The damping time scale of the perturbation is proportional to the black hole mass, and it is shorter for higher-order modes $\left(\omega_{i, n+1}>\omega_{i, n}\right)$. Thus the detection of gravitational wave emitted from a perturbed black hole can be used to directly measure the black hole mass.

(d) Some properties of quasinormal frequencies can be

\begin{tabular}{|c|c|c|}
\hline $\mathrm{n} \mid \ell=2$ & $\ell=3$ & $\ell=4$ \\
\hline \begin{tabular}{l|lll}
0 & 0.37367 & $-0.08896 i$
\end{tabular} & $0.59944-0.09270 \mathrm{i}$ & $0.80918-0.09416 \mathrm{i}$ \\
\hline $0.34671-0.27391 \mathrm{i}$ & $0.58264-0.28130 \mathrm{i}$ & $0.79663-0.28443 \mathrm{i}$ \\
\hline $0.30105-0.47828 \mathrm{i}$ & $0.55168-0.47909 \mathrm{i}$ & $0.77271-0.47991 \mathrm{i}$ \\
\hline $0.25150-0.70514 \mathrm{i}$ & $0.51196-0.69034 \mathrm{i}$ & $0.73984-0.68392 \mathrm{i}$ \\
\hline
\end{tabular}
learned from the following table

We learned that for the same $l$, with the increase of $n$, the real part of quasinormal frequencies decreases, while the
TABLE I: The first four QNM frequencies ( $\omega M)$ of the Schwarzschild black hole for $\ell=2,3$, and 4[3].

imaginary part increases. This corresponds to say that for the higher modes, the perturbation will have less oscillations outside of the black hole and die out quicker. With the increase of the multipole index $l$, we found that both real part and imaginary part of quasinormal frequencies increase, which shows that for the bigger $l$ the perturbation outside the black hole will oscillate more but die out quicker in the asymptotically flat spacetimes. This property will change if one studies the AdS and dS spacetimes, since the behavior of the effective potential will be changed there.

All previous works on quasinormal modes have so far been restricted to time-independent black hole backgrounds. It should be realized that, for a realistic model, the black hole parameters change with time. A black hole gaining or losing mass via absorption (merging) or evaporation is a good example. The more intriguing investigation of the black hole quasinormal modes calls for a systematic analysis of timedependent spacetimes. Recently the late time tails under the influence of a time-dependent scattering potential has been explored in [22], where the tail structure was found to be modified due to the temporal dependence of the potential. The 


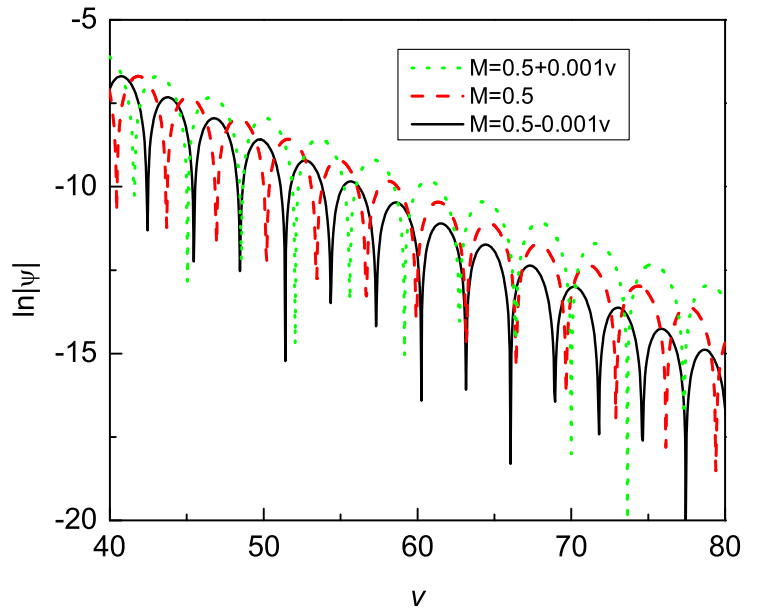

FIG. 1: Temporal evolution of the field in the background of Vaidya metric $(q=0)$ for $l=2$, evaluated at $r=5$. The mass of the black hole is $M(v)=0.5 \pm 0.001 v$. The field evolution for $M(v)=0.5+0.001 v$ and $M(v)=0.5-0.001 v$ are shown as the top curve and the bottom curve respectively. For comparison, the oscillations for $M=0.5$ is given in the middle line.

exploration on the modification to the quasinormal modes in time-dependent spacetimes has also been started. Instead of plotting an effective time-dependent scattering potential by hand as done in [22], we have introduced the time-dependent potential in a natural way by considering dynamical black holes, with black hole parameters changing with time due to absorption and evaporation processes. We have studied the temporal evolution of massless scalar field perturbation [23][24].

We found that the modification to the QNMs due to the time-dependent background is clear. When the black hole mass $M$ increases linearly with time, the decay becomes slower compared to the stationary case, which corresponds to saying that $\left|\omega_{i}\right|$ decreases with respect to time. The oscillation period is no longer a constant as in the stationary Schwarzschild black hole. It becomes longer with the increase of time. In other words, the real part of the quasinormal frequency $\omega_{r}$ decreases with the increase of time. When $M$ decreases linearly with respect to time, compared to the stationary Schwarzschild black hole, we have observed that the decay becomes faster and the oscillation period becomes shorter, thus both $\left|\omega_{i}\right|$ and $\omega_{r}$ increase with time. The objective picture can be seen in Fig.1.

\section{PERTURBATIONS IN ADS AND DS BLACK HOLE SPACETIMES}

Motivated by the recent discovery of the Anti-de Sitter/Conformal Field Theory (AdS/CFT) correspondence, the investigation of QNMs in Anti-de Sitter (AdS) spacetimes became appealing in the past years. It was argued that the QNMs of AdS black holes have direct interpretation in terms of the dual conformal field theory.

The first study of the QNMs in AdS spaces was performed by Chan and Mann [4]. Subsequently, Horowitz and Hubeny suggested a numerical method to calculate the QN frequencies directly and made a systematic investigation of QNMs for scalar perturbation on the background of Schwarzschild AdS (SAdS) black holes [5]. They claimed that for large AdS black holes both the real and imaginary parts of the quasinormal frequencies scale linearly with the black hole temperature. However for small AdS black holes they found a departure from this behaviour. This was further confirmed by the object picture obtained in [12].

Considering that the Reissner-Nordstrom AdS (RNAdS) black hole solution provides a better framework than the SAdS geometry and may contribute significantly to our understanding of space and time, the Horowitz-Hubeny numerical method was generalized to the study of QNMs of RNAdS black holes in [6] and later crosschecked by using the time evolution approach [7]. Unlike the SAdS case, the quasinormal frequencies do not scale linearly with the black hole temperature, and the approach to thermal equilibrium in the CFT was more rapid as the charge on the black hole increased. In addition to the scalar perturbation, gravitational and electromagnetic perturbations in AdS black holes have also attracted attention [8, 9]. Other works on QNMs in AdS spacetimes can be found in [10-15]. Recently in [9] Berti and Kokkotas used the frequency-domain method and restudied the scalar perturbation in RNAdS black holes. They verified most of our previous numerical results in $[6,7]$.

As was pointed out in [6] and later supported in [9], the Horowitz-Hubeny method breaks down for large values of the charge. To study the QNMs in the near extreme and extreme RNAdS backgrounds, we need to count on time evolution approach. Employing an improved numerical method, we have shown that the problem with minor instabilities in the form of "plateaus", which were observed in [7], can be overcome. We obtained the precise QNMs behavior in the highly charged RNAdS black holes.

To illustrate the properties of quasinormal modes in AdS black holes, we here briefly review the perturbations around RNAdS black holes. The metric describing a charged, asymptotically Anti-de Sitter spherical black hole, written in spherical coordinates, is given by

$$
d s^{2}=-h(r) d t^{2}+h(r)^{-1} d r^{2}+r^{2}\left(d \theta^{2}+\sin ^{2} \theta d \phi^{2}\right),
$$

where the function $h(r)$ is

$$
h(r)=1-\frac{2 m}{r}+\frac{Q^{2}}{r^{2}}-\frac{\Lambda r^{2}}{3}
$$

We are assuming a negative cosmological constant, usually written as $\Lambda=-3 / R^{2}$. The integration constants $m$ and $Q$ are the black hole mass and electric charge respectively. The extreme value of the black hole charge, $Q_{\max }$, is given by the function of the event horizon radius in the form $Q_{\max }^{2}=r_{+}^{2}(1+$ $\left.3 r_{+}^{2} / R^{2}\right)$. Consider now a scalar perturbation field $\Phi$ obeying the massless Klein-Gordon equation 


$$
\square \Phi=0 .
$$

The usual separation of variables in terms of a radial field and a spherical harmonic $\mathrm{Y}_{\ell, m}(\theta, \varphi)$,

$$
\Phi=\sum_{\ell m} \frac{1}{r} \Psi(t, r) \mathrm{Y}_{\ell m}(\theta, \phi)
$$

leads to Schrödinger-type equations in the tortoise coordinate for each value of $\ell$. Introducing the tortoise coordinate $r^{*}$ by $\frac{d r^{*}}{d r}=h(r)^{-1}$ and the null coordinates $u=t-r^{*}$ and $v=t+r^{*}$, the field equation can be written as

$$
-4 \frac{\partial^{2} \Psi}{\partial u \partial v}=V(r) \Psi
$$

where the effective potential $V$ is

$$
V(r)=h(r)\left[\frac{\ell(\ell+1)}{r^{2}}+\frac{2 m}{r^{3}}-\frac{2 Q^{2}}{r^{4}}+\frac{2}{R^{2}}\right] .
$$

Wave equation (10) is useful to study the time evolution of the scalar perturbation, in the context of an initial characteristic value problem.
In terms of the ingoing Eddington coordinates $(v, r)$ and separating the scalar field in a product form as

$$
\Phi=\sum_{\ell m} \frac{1}{r} \psi(r) \mathrm{Y}_{\ell m}(\theta, \phi) e^{-i \omega v}
$$

the minimally-coupled scalar wave equation (8) may thereby be reduced to

$$
h(r) \frac{\partial^{2} \psi(r)}{\partial r^{2}}+\left[h^{\prime}(r)-2 i \omega\right] \frac{\partial \psi(r)}{\partial r}-\tilde{V}(r) \psi(r)=0
$$

where $\tilde{V}(r)=V(r) / h(r)=h^{\prime}(r) / r+\ell(\ell+1) / r^{2}$.

Introducing $x=1 / r$, Eq.(13) can be re-expressed as Eqs.(15-18) in [6]. These equations are appropriate to directly obtain the QN frequencies using the Horowitz-Hubeny method.

We have used two different numerical methods to solve the wave equations. The first method is the Horowitz-Hubeny method. The second numerical methods we have employed is the discretization for equation (10) in the form

$$
\left[1-\frac{\Delta^{2}}{16} V(S)\right] \psi(N)=\psi(E)+\psi(W)-\psi(S)-\frac{\Delta^{2}}{16}[V(S) \psi(S)+V(E) \psi(E)+V(W) \psi(W)]
$$

The points $N, S, W$ and $E$ are defined as usual: $N=(u+\Delta, v+$ $\Delta), W=(u+\Delta, v), E=(u, v+\Delta)$ and $S=(u, v)$. The local truncation error is of the order of $O\left(\Delta^{4}\right)$.

Figure 2 demonstrates the behaviors of the field with the increase of the charge in RN AdS black hole background. Since the imaginary and real parts of the quasinormal frequencies relate to the damping time scale $\left(\tau_{1}=1 / \omega_{i}\right)$ and oscillation time scale $\left(\tau_{2}=1 / \omega_{r}\right)$, respectively. We learned that as $Q$ increases $\omega_{i}$ increases as well, which corresponds to the decrease of the damping time scale. According to the AdS/CFT correspondence, this means that for bigger $Q$, it is quicker for the quasinormal ringing to settle down to thermal equilibrium. From figure 2 it is easy to see this property. Besides, figure 2 also tells us that the bigger $Q$ is, the lower frequencies of oscillation will be. If we perturb a RN AdS black hole with high charge, the surrounding geometry will not "ring" as much and as long as that of the black hole with small $Q$. It is easy for the perturbation on the highly charged AdS black hole background to return to thermal equilibrium.

However this relation seems not to hold well when the charge is sufficiently big. We see that over some critical value of $Q$, the damping time scale increases with the increase of $Q$, corresponding to the decrease of imaginary frequency. This means that over some critical value of $Q$, the larger the black hole charge is, the slower for the outside per- turbation to die out. Besides the oscillation starts to disappear at $Q_{c}=0.3895 Q_{\max }$. These points can be directly seen in the wave function plotting from Fig. 3 and Fig.4.

In addition to the study of the lowest lying QNMs, it is interesting to study the higher overtone QN frequencies for scalar perturbations. The first attempt was carried out in [15]. We have extended the study to the RNAdS backgrounds. It was argued that the dependence of the QN frequencies on the angular index $\ell$ is extremely weak [9]. This was also claimed in [15]. Using our numerical results we have shown that this weak dependence on the angular index is not trivial.

For the same value of the charge, we have found that both real and imaginary parts of QN frequencies increase with the overtone number $n$, which is different from that observed in asymptotically flat spacetimes [25] where $\omega_{r}$ approaches a constant while $\omega_{i}$ tends to infinity in the limit $n \rightarrow \infty$. In asymptotically flat spacetimes, the constant $\omega_{r}$ was claimed as just the right one to make loop quantum gravity give the correct result for the black hole entropy with some (not clear yet) correspondence between classical and quantum states. However such correspondence seems do not hold in AdS space.

For the large black hole regime the frequencies become evenly spaced for high overtone number $n$. For lowly charged RNAdS black hole, choosing bigger values of the charge, the real part in the spacing expression becomes smaller, while the 


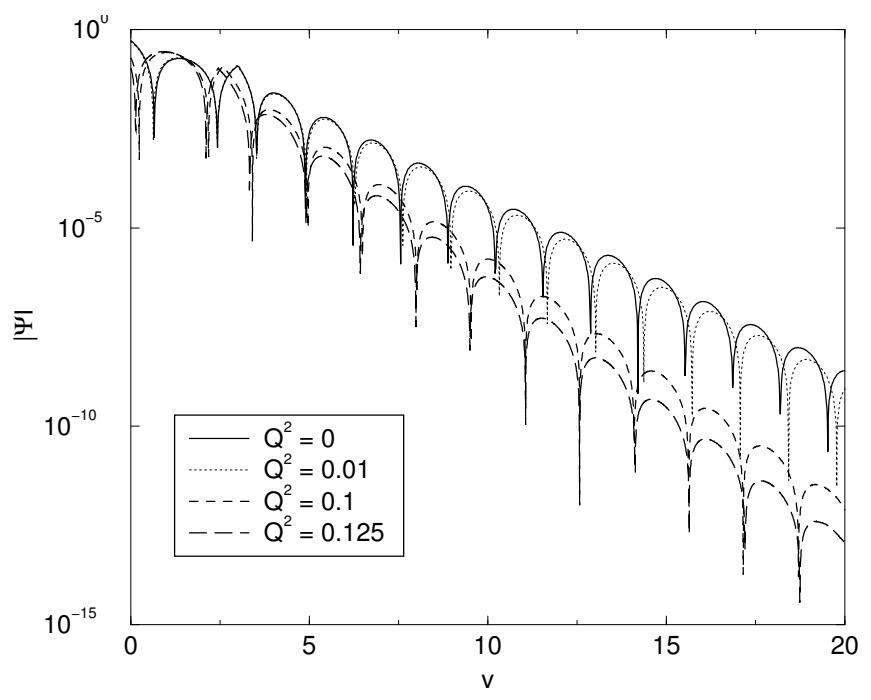

FIG. 2: Semi-log graphs of $|\Psi|$ with $r_{+}=0.4$ and small values of $Q$. The extreme value for $Q^{2}$ is 0.2368 .
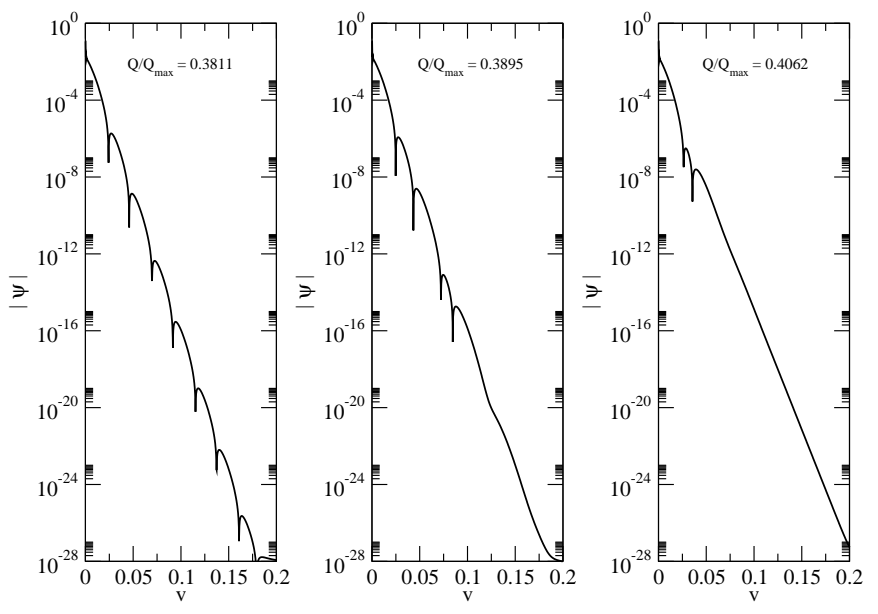

FIG. 3: Semi-log graphs of the scalar field in the event horizon, showing the transition from oscillatory to non-oscillatory asymptotic decay. In the graphs, $r_{+}=100, \ell=0$ and $R=1$.

imaginary part becomes bigger. This calls for further understanding from CFT.

Qualitative correspondences between quasinormal modes in AdS spaces and the decay of perturbations in the due CFT have been obtained above, while quantitive examinations are difficult to be done in usual four-dimensional black holes due to the mathematical complicacy. Encouragingly, in the threedimensional (3D) BTZ black hole model [26], the mathematical simplicity can help us to understand the problem much better. A precise quantitative agreement between the quasinormal frequencies and the location of poles of the retarded correlation function of the corresponding perturbations in the dual CFT has been presented [27]. This gives a further evidence of the correspondence between gravity in AdS spacetime and quantum field theory at the boundary.

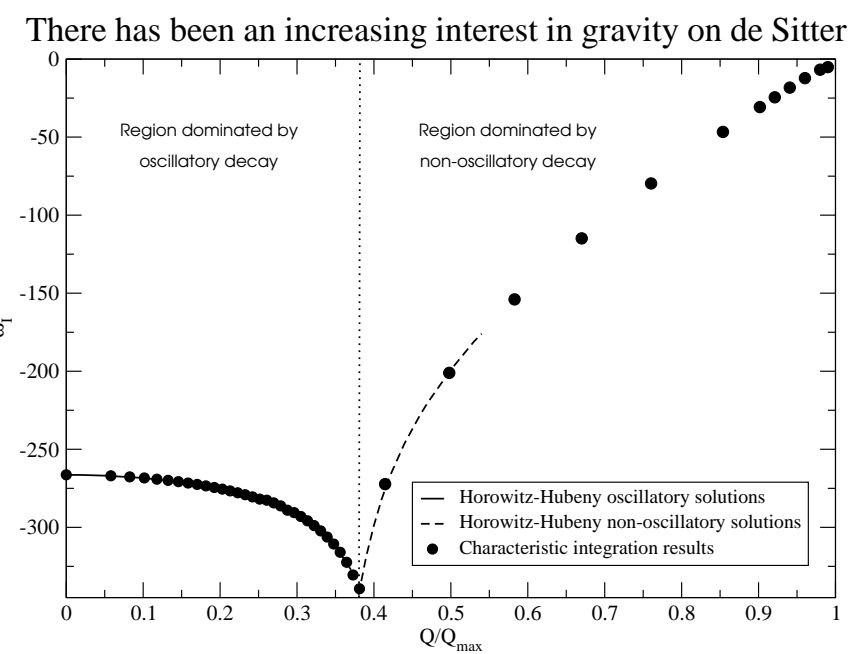

FIG. 4: Graph of $\omega_{i}$ with $Q / Q_{\max }$, showing that $\omega_{i}$ tends to zero as $Q$ tends to $Q_{\max }$. In the graph, $r_{+}=100, R=1, \ell=0$ and $n=0$.

(dS) spacetimes in view of recent observational support for a positive cosmological constant. A holographic duality relating quantum gravity on D-dimensional dS space to CFT on (D-1)-sphere has been proposed [28]. It is of interest to extend the study in [27] to dS space by displaying the exact solution of the quasinormal mode problem in the dS bulk space and exploring its relation to the CFT theory at the boundary. This could serve as a quantitative test of the dS/CFT correspondence. We have used the nontrivial 3D dS spacetimes as a testing ground to examine the $\mathrm{dS} / \mathrm{CFT}$ correspondence [18][19]. The mathematical simplicity in these models renders all computations analytical.

The metric of the $3 \mathrm{D}$ rotating $\mathrm{dS}$ spacetime is given by

$$
d s^{2}=-\left(M-\frac{r^{2}}{l^{2}}+\frac{J^{2}}{4 r^{2}}\right) d t^{2}+\left(M-\frac{r^{2}}{l^{2}}+\frac{J^{2}}{4 r^{2}}\right)^{-1} d r^{2}+r^{2}\left(d \varphi-\frac{J}{2 r^{2}} d t\right)^{2}
$$

where $J$ is associated to the angular momentum. The horizon

of such spacetime can be obtained from

$$
M-\frac{r^{2}}{l^{2}}+\frac{J^{2}}{4 r^{2}}=0
$$


The solution is given in terms of $r_{+}$and $-i r_{-}$, where $r_{+}$corresponds to the cosmological horizon and $-i r_{-}$here being imaginary, has no physical interpretation in terms of a horizon. Using $r_{+}$and $r_{-}$, the mass and angular momentum of spacetime can be expressed as

$$
M=\frac{r_{+}^{2}-r_{-}^{2}}{l^{2}}, \quad J=\frac{-2 r_{+} r_{-}}{l}
$$

Scalar perturbations of this spacetime are described by the wave equation

$$
\frac{1}{\sqrt{-g}} \partial_{\mu}\left(\sqrt{-g} g^{\mu \nu} \partial_{\nu} \Phi\right)-\mu^{2} \Phi=0
$$

where $\mu$ is the mass of the field. Adopting the separation

$$
\Phi(t, r, \varphi)=R(r) e^{-i \omega t} e^{i m \varphi},
$$

the radial part of the wave equation can be written as

$$
\begin{aligned}
\frac{1}{g_{r r}} \mu^{2} R= & \frac{1}{g_{r r}} \frac{d}{r d r}\left(\frac{r^{2}}{g_{r r}} \frac{d R}{r d r}\right)+\left[\omega^{2}-\frac{1}{r^{2}} m^{2}(M-\right. \\
& \left.\left.\frac{r^{2}}{l^{2}}\right)-\frac{J}{r^{2}} m \omega\right] R,
\end{aligned}
$$

where $g_{r r}=\left(M-r^{2} / l^{2}+J^{2} /\left(4 r^{2}\right)\right)^{-1}$. Employing (17) and defining $z=\frac{r^{2}-r_{+}^{2}}{r^{2}-\left(-i r_{-}\right)^{2}}$, the radial wave equation can be simplified into

$$
(1-z) \frac{d}{d z}\left(z \frac{d R}{d z}\right)+\left[\frac{1}{z}\left(\frac{\omega l^{2} r_{+}+m l r_{-}}{2\left(r_{+}^{2}+r_{-}^{2}\right)}\right)^{2}-\left(\frac{-\omega l^{2} i r_{-}+i m l r_{+}}{2\left(r_{+}^{2}+r_{-}^{2}\right)}\right)^{2}+\frac{1}{4(1-z)} \mu^{2} l^{2}\right] R=0
$$

We now set the Ansatz

$$
R(z)=z^{\alpha}(1-z)^{\beta} F(z)
$$

and Eq.(21) can be transformed into

$$
\begin{aligned}
z(1-z) \frac{d^{2} F}{d z^{2}}+ & {[1+2 \alpha-(1+2 \alpha+2 \beta) z] \frac{d F}{d z}+\left\{\left(\beta(\beta-1)+\frac{\mu^{2} l^{2}}{4}\right) \frac{1}{1-z}+\frac{1}{z}\left[\left(\frac{\omega l^{2} r_{+}+m l r_{-}}{2\left(r_{+}^{2}+r_{-}^{2}\right)}\right)^{2}+\alpha^{2}\right]\right.} \\
- & {\left.\left[\left(\frac{-i \omega l^{2} r_{-}+i m l r_{+}}{2\left(r_{+}^{2}+r_{-}^{2}\right)}\right)^{2}+\alpha^{2}+(1+2 \alpha) \beta+\beta(\beta-1)\right]\right\} F=0 . }
\end{aligned}
$$

Comparing with the standard hypergeometric equation

$$
z(1-z) \frac{d^{2} F}{d z^{2}}+[c-(1+a+b) z] \frac{d F}{d z}-a b F=0
$$

we have

$$
\begin{aligned}
c & =1+2 \alpha \\
a+b & =2 \alpha+2 \beta \\
\alpha^{2}+\left(\frac{\omega l^{2} r_{+}+m l r_{-}}{2\left(r_{+}^{2}+r_{-}^{2}\right)}\right)^{2} & =0 \\
\beta(\beta-1)+\frac{\mu^{2} l^{2}}{4} & =0 \\
\left(\frac{-\omega l^{2} i r_{-}+i m l r_{+}}{2\left(r_{+}^{2}+r_{-}^{2}\right)}\right)^{2}+(\alpha+\beta)^{2} & =a b
\end{aligned}
$$

Without loss of generality, we can take

$$
\begin{aligned}
\alpha & =-i\left(\frac{\omega l^{2} r_{+}+m l r_{-}}{2\left(r_{+}^{2}+r_{-}^{2}\right)}\right), \\
\beta & =\frac{1}{2}\left(1-\sqrt{1-\mu^{2} l^{2}}\right),
\end{aligned}
$$

which leads to

$$
\begin{aligned}
& a=-\frac{i}{2}\left(\frac{\omega l^{2}+i m l}{r_{+}+i r_{-}}+i\left(1-\sqrt{1-\mu^{2} l^{2}}\right)\right), \\
& b=-\frac{i}{2}\left(\frac{\omega l^{2}-i m l}{r_{+}-i r_{-}}+i\left(1-\sqrt{1-\mu^{2} l^{2}}\right)\right), \\
& c=1-i\left(\frac{\omega l^{2} r_{+}+m l r_{-}}{r_{+}^{2}+r_{-}^{2}}\right),
\end{aligned}
$$


and the solution of (21) reads

$$
R(z)=z^{\alpha}(1-z)_{2}^{\beta} F_{1}(a, b, c, z) .
$$

Using basic properties of the hypergeometric equation we write the result as

$$
\begin{aligned}
R(z) & =z^{\alpha}(1-z)^{\beta}(1-z)^{c-a-b} \frac{\Gamma(c) \Gamma(a+b-c)}{\Gamma(a) \Gamma(b)}{ }_{2} F_{1}(c-a, c-b, c-a-b+1,1-z) \\
& +z^{\alpha}(1-z)^{\beta} \frac{\Gamma(c) \Gamma(c-a-b)}{\Gamma(c-a) \Gamma(c-b)}{ }_{2} F_{1}(a, b, a+b-c+1,1-z)
\end{aligned}
$$

The first term vanishes at $z=1$, while the second vanishes provided that

$$
c-a=-n, \quad \text { or } \quad c-b=-n,
$$

where $n=0,1,2, \ldots$ Employing Eqs (27), it is easy to see that the quasinormal frequencies are

$$
\begin{aligned}
& \omega_{R}=i \frac{m}{l}-2 i\left(\frac{r_{+}-i r_{-}}{l^{2}}\right)\left(n+\frac{h_{+}}{2}\right) \\
& \omega_{L}=-i \frac{m}{l}-2 i\left(\frac{r_{+}+i r_{-}}{l^{2}}\right)\left(n+\frac{h_{+}}{2}\right),
\end{aligned}
$$

where $h_{ \pm}=1 \pm \sqrt{1-\mu^{2} l^{2}}$. Taking other values of $\alpha$ and $\beta$ satisfying (25), we have also the frequencies

$$
\begin{aligned}
& \omega_{R}=i \frac{m}{l}+2 i\left(\frac{r_{+}-i r_{-}}{l^{2}}\right)\left(n+\frac{h_{+}}{2}\right) \\
& \omega_{L}=-i \frac{m}{l}+2 i\left(\frac{r_{+}+i r_{-}}{l^{2}}\right)\left(n+\frac{h_{+}}{2}\right), \\
& \omega_{R}=i \frac{m}{l}-2 i\left(\frac{r_{+}-i r_{-}}{l^{2}}\right)\left(n+\frac{h_{-}}{2}\right) \\
& \omega_{L}=-i \frac{m}{l}-2 i\left(\frac{r_{+}+i r_{-}}{l^{2}}\right)\left(n+\frac{h_{-}}{2}\right), \\
& \omega_{R}=i \frac{m}{l}+2 i\left(\frac{r_{+}-i r_{-}}{l^{2}}\right)\left(n+\frac{h_{-}}{2}\right) \\
& \omega_{L}=-i \frac{m}{l}+2 i\left(\frac{r_{+}+i r_{-}}{l^{2}}\right)\left(n+\frac{h_{-}}{2}\right) .
\end{aligned}
$$

For a thermodynamical system the relaxation process of a small perturbation is determined by the poles, in the momentum representation, of the retarded correlation function of the perturbation. Let's now investigate the quasinormal modes from the CFT side. Define the invariant distance between two points defined by $x$ and $x^{\prime}$ reads [28][29]

$$
d=l \arccos P
$$

where $P=X^{A} \eta_{A B} X^{\prime B}$. In the limit $r, r^{\prime} \rightarrow \infty$,

$$
\begin{aligned}
P & \approx 2 \sinh \frac{\left(i r_{+}+r_{-}\right)(l \Delta \varphi-i \Delta t)}{2 l^{2}} \\
& \times \sinh \frac{\left(i r_{+}-r_{-}\right)(l \Delta \varphi+i \Delta t)}{2 l^{2}} .
\end{aligned}
$$

This means that we can find the Hadamard Green's function as defined by [29] in terms of $P$. Such a Green's function is defined as $G\left(u, u^{\prime}\right)=<0\left|\left\{\phi(u), \phi\left(u^{\prime}\right)\right\}\right| 0>$ with $\left(\nabla_{x}^{2}-\mu^{2}\right) G=$ 0 . It is possible to obtain the solution

$$
G \sim F\left(h_{+}, h_{-}, 3 / 2,(1+P) / 2\right)
$$

in the limit $r, r^{\prime} \rightarrow \infty$.

Following [28] [29], we choose boundary conditions for the fields such that

$$
\lim _{r \rightarrow \infty} \phi(r, t, \varphi) \rightarrow r^{-h_{-}} \phi_{-}(t, \varphi)
$$

Then, for large $r, r^{\prime}$, an expression for the two point function of a given operator $O$ coupling to $\phi$ has the form

$$
\int d t d \varphi d t^{\prime} d \varphi^{\prime} \frac{\left(r r^{\prime}\right)^{2}}{l^{2}} \phi \overleftrightarrow{\partial_{r *}} G \overleftrightarrow{\partial_{r *}} \phi=\int d t d \varphi d t^{\prime} d \varphi^{\prime} \phi \frac{1}{\left[2 \sinh \frac{\left(i r_{+}+r_{-}\right)(l \Delta \varphi-i \Delta t)}{2 l^{2}} \sinh \frac{\left(i r_{+}-r_{-}\right)(l \Delta \varphi+i \Delta t)}{2 l^{2}}\right]^{h_{+}}} \phi
$$

where $r *$ in (39) is the tortoise coordinate. 


$$
\begin{aligned}
& \int d t d \varphi d t^{\prime} d \varphi^{\prime} \frac{\exp \left(-i m^{\prime} \varphi^{\prime}-i \omega^{\prime} t^{\prime}+i m \varphi+i \omega t\right)}{\left[2 \sinh \frac{\left(i r_{+}+r_{-}\right)(l \Delta \varphi-i \Delta t)}{2 l^{2}} \sinh \frac{\left(i r_{+}-r_{-}\right)(l \Delta \varphi+i \Delta t)}{2 l^{2}}\right]^{h_{+}}} \\
& \approx \delta_{m m^{\prime}} \delta\left(\omega-\omega^{\prime}\right) \Gamma\left(h_{+} / 2+\frac{i m / 2 l+\omega / 2}{2 \pi T}\right) \Gamma\left(h_{+} / 2-\frac{i m / 2 l+\omega / 2}{2 \pi T}\right) \Gamma\left(h_{+} / 2+\frac{i m / 2 l-\omega / 2}{2 \pi \bar{T}}\right) \\
& \times \Gamma\left(h_{+} / 2-\frac{i m / 2 l-\omega / 2}{2 \pi \bar{T}}\right)
\end{aligned}
$$

where we changed variables to $v=l \varphi+i t, \bar{v}=l \varphi-i t$, and $T=\frac{i r_{+}-r_{-}}{2 \pi l^{2}}, \bar{T}=\frac{i r_{+}+r_{-}}{2 \pi l^{2}}$. The poles of such a correlator are

$$
\begin{aligned}
& \omega_{L}=-\frac{i m}{l} \pm 2 \frac{i r_{+}-r_{-}}{l^{2}}\left(n+h_{+} / 2\right), \\
& \omega_{R}=\frac{i m}{l} \pm 2 \frac{i r_{+}+r_{-}}{l^{2}}\left(n+h_{+} / 2\right),
\end{aligned}
$$

corresponding to the quasinormal modes $(31,34)$ obtained before.

The quasinormal eigenfunctions thus correspond to excitation of the corresponding CFT, being exactly those that appear in the spectrum of the two point functions of CFT operators in dS background for large values of $r$, that is at the boundary.

The poles of such a correlator correspond exactly to the QNM's obtained from the wave equation in the bulk. This provides a quantitative test of the dS/CFT correspondence. This work has been extended to four-dimensional dS spacetimes [19].

\section{CONCLUSIONS AND OUTLOOKS}

Perturbations around black holes have been a hot topic in general relativity in the last decades. The main reason is its astrophysical importance of the study in order to foresee gravitational waves. To extract as much information as possible from the future gravitational wave observations, the accurate quasinormal modes waveforms are needed for different kinds of black holes, including different stationary black holes (especially interesting with rotations), time-dependent black hole spacetimes which can describe the black hole absorption and evaporation processes and extremely interesting situations with colliding black holes since they may produce stronger gravitational wave signals which may be easier to be detected.

Besides astronomical interest, perturbations around black holes can also serve as a testing ground to examine the recent theories proposed in string theory, such as the relation between physics in (A)dS spaces and Conformal Field Theory on its boundary. Very recently, it was even argued that quasinormal modes could be a way to detect extra dimensions. String theory makes the radial prediction that spacetime has extra dimensions and gravity propagates in higher dimensions. Using the black string model as an example, it was shown that different from the late time signal-simple power-law tail in the usual four-dimensions, high frequency signal persists in the black string [20]. These frequencies are characters of the massive modes contributed from extra dimensions. This possible way to detect extra-dimensions needs further theoretical examinations and it is expected that future gravitational wave observation could help to prove the existence of the extra dimensions so that can give support to the fundamental string theory.

\section{Acknowledgments}

This work was supported by NNSF of China, Ministry of Education of China, Shanghai Science and Technology Commission and Shanghai Education Commission. We acknowledge many helpful discussions with C. Y. Lin, C. Molina and E. Abdalla.
[1] Zhi-Qiang Shen, K. Y. Lo, M.-C. Liang, Paul T. P. Ho and J.-H. Zhao, Nature, 438, 1038 (2005).

[2] H. P. Nollert, Class. Quant. Grav. 16, R159 (1999).

[3] K. D. Kokkotas and B. G. Schmidt, Living Rev. Rel. 2, 2 (1999).

[4] J. S. F. Chan and R. B. Mann, Phys. Rev. D 55, 7546 (1997); J.S.F. Chan and R.B. Mann, Phys. Rev. D 59, 064025 (1999).

[5] G. T. Horowitz and V. E. Hubeny, Phys. Rev. D 62, 024027 (2000).

[6] B. Wang, C.Y. Lin, and E. Abdalla, Phys. Lett. B 481, 79 (2000).

[7] B. Wang, C. Molina, and E. Abdalla, Phys. Rev. D 63, 084001 (2001).
[8] V. Cardoso and J.P.S. Lemos, Phys. Rev. D 63, 124015 (2001); V. Cardoso and J.P.S. Lemos, Phys. Rev. D 64, 084017 (2001); V. Cardoso and J.P.S. Lemos, Class. Quantum Grav. 18, 5257 (2001).

[9] E. Berti and K.D. Kokkotas, Phys. Rev. D 67, 064020 (2003).

[10] R. A. Konoplya, Phys. Rev. D 66, 044009 (2002).

[11] D. Birmingham, I. Sachs, S. N. Solodukhin, Phys. Rev. Lett. 88, 151301 (2002); D. Birmingham, Phys.Rev. D 64, 064024 (2001).

[12] J.M. Zhu, B. Wang, and E. Abdalla, Phys. Rev. D 63, 124004 (2001); B. Wang, E. Abdalla and R. B. Mann, Phys. Rev. D 65, 084006 (2002). 
[13] S. Musiri, G. Siopsis, Phys. Lett. B 576, 309-313 (2003); R. Aros, C. Martinez, R. Troncoso, J. Zanelli, Phys. Rev. D 67, 044014 (2003); A. Nunez, A. O. Starinets, Phys.Rev. D 67,124013 (2003); E. Winstanley, Phys. Rev. D 64, 104010 (2001); V. Cardoso, J. Natario and R. Schiappa, hepth/0403132; R.A. Konoplya, Phys.Rev.D 66, 084007 (2002).

[14] V. Cardoso and J. P. S. Lemos, Phys. Rev. D 67, 084020 (2003).

[15] V. Cardoso, R. Konoplya and J. P. S. Lemos, Phys. Rev. D 68 044024 (2003)

[16] P. R. Brady, C. M. Chambers, W. Krivan and P. Laguna, Phys. Rev. D 55, 7538 (1997); P. R. Brady, C. M. Chambers, W. G. Laarakkers and E. Poisson, Phys. Rev. D 60, 064003 (1999); T.Roy Choudhury, T. Padmanabhan, Phys. Rev. D 69064033 (2004); D. P. Du, B. Wang and R. K. Su, Phys.Rev. D70 (2004) 064024, hep-th/0404047.

[17] C. Molina, Phys.Rev. D 68 (2003) 064007; C. Molina, D. Giugno, E. Abdalla, A. Saa, Phys.Rev. D 69 (2004) 104013.

[18] E. Abdalla, B. Wang, A. Lima-Santos and W. G. Qiu, Phys. Lett. B 538, 435 (2002).

[19] E. Abdalla, K. H. Castello-Branco and A. Lima-Santos, Phys. Rev. D 66, 104018 (2002)
[20] Sanjeev S. Seahra, Chris Clarkson, Roy Maartens, Phys.Rev.Lett. 94 (2005) 121302; Chris Clarkson, Roy Maartens, astro-ph/0505277.

[21] S. Hod, Phys. Rev. D 58, 104022 (1998); 61, 024033(2000); 61, 064018 (2000); L. Barack and A. Ori, Phys. Rev. Lett. 82, 4388 (1999); Phys. Rev. D 60, 124005 (1999); N. Andersson and K. Glampedakis, Phys. Rev. Lett. 84, 4537 (2000).

[22] S. Hod, Phys. Rev. D 66, 024001 (2002).

[23] L.H. Xue, Z.X. Shen, B. Wang and R.K. Su, Mod. Phys. Lett. A 19, 239(2004).

[24] Cheng-Gang Shao, Bin Wang, Elcio Abdalla, Ru-Keng Su, Phys.Rev. D71 (2005) 044003.

[25] Shahar Hod, Phys.Rev.Lett. 81 (1998) 4293.

[26] M. Banados, C. Teitelboim and J. Zanelli, Phys. Rev. Lett. 69, 1849 (1992).

[27] D. Birmingham, I. Sachs and S. N. Solodukhin, Phys. Rev. Lett 88151301 (2002), hep-th/0112055.

[28] A. Strominger JHEP 0110 (2001) 034, hep-th/0106113.

[29] D. Klemm, Nucl. Phys. B625 (2002) 295-311, hep-th/0106247. 